\title{
The meaning of 'concentration'
}

\author{
René Dybkaer
}

Received: 3 September 2007 / Accepted: 21 September 2007/Published online: 3 November 2007

(C) Springer-Verlag 2007

\begin{abstract}
Terminology within a small laboratory community can be informal without danger of misunderstanding, but communication with a wider audience and not least in publications, needs unequivocal terms for defined concepts. As an example, the many meanings of "concentration" are explored to present a systematic nomenclature, including a concept diagram.
\end{abstract}

Keywords Concentration - Concept system .

Kind-of-quantity $\cdot$ Systematic terminology

\section{Introduction}

In colloquial English, the phrase 'concentrating on ...' indicates focussing the mind on an undertaking whereas the phrase 'concentration of ...' refers to grouping of entities. ${ }^{1}$

In many scientific disciplines, the latter phrase is used in two senses:

1. the process of increasing the amount (in the colloquial sense, not specifically amount-of-substance) of a component of interest in a volume of mixture, e.g., by selective evaporation of solvent;

2. the state of a material, either as amount of a given component related to amount of surrounding matrix or amount of component related to total amount of material.

Here, only the many quantities describing the state of materials will be discussed with a view to a systematic

R. Dybkaer $(\bowtie)$

Department of Standardization in Laboratory Medicine,

REGION H Frederiksberg Hospital,

Copenhagen University Hospital, 2000 Frederiksberg, Denmark nomenclature, based on various International Standards [1] and recommendations [2-7].

\section{Conventions in this paper}

The following concepts with abbreviations or symbols and definitions will be used in this text:

$\mathrm{S}$

system: part or phenomenon of the perceivable or conceivable world consisting of a demarcated arrangement of a set of elements and a set of relationships or processes between these elements [5-3.3], i.e., occurrence, body, substance, mixture, or material carrying or embodying a property or a quantity, e.g., light beam; sample of wine;

$\mathrm{B}, \mathrm{C}, . ., \mathrm{N}$

component: part of a system [5-3.4], e.g., sodium ions in sea water, ozone in air, erythrocytes in blood; often called analyte (but unfortunately this term is also incorrectly used for the entire combination of 'system, component, and kind-of-quantity' constituting the representation of the concept "quantity");

A

major component: component that is a greater part, e.g., solvent or matrix;

$q$

quantity: property of a phenomenon, body, or substance, where the property has a magnitude that can be expressed as a number and a reference [7-1.1], e.g., mass of glucose in a given bottle of orange juice;

\footnotetext{
${ }^{1}$ In this paper, single quotation marks '...' indicate a term or a quotation, double quotation marks “...” indicate a concept when necessary.
} 
$Q$

kind-of-quantity: aspect common to mutually comparable quantities [7-1.2], e.g., length, amount-of-substance concentration; often simply called quantity.

\section{Specific concepts under kind-of-quantity}

A kind-of-quantity used in physics, chemistry, and biology is either:

- a base kind-of-quantity that is conventionally chosen and has a corresponding base unit, e.g., mass $m$, amount-of-substance $n$; or

- a derived kind-of-quantity defined by a quantity equation, e.g., volume equals cubed length, $V=l^{3}$, volumic mass (= mass density) equals mass of system divided by volume of system, $\rho_{\mathrm{S}}=m_{\mathrm{S}} / V_{\mathrm{S}}$.

A kind-of-quantity is also either

- extensive, i.e., comprising individual quantities having values varying with sizes of system of constant composition, e.g., time, number of entities, or

- intensive, i.e., comprising individual quantities having values independent of size of a system of constant composition, e.g., number concentration.

The concept of "intensive kind-of-quantity" may be divided into:

- "material kind-of-quantity", defined as a ratio between a numerator and a denominator extensive kind-ofquantity, both involving the same system or the same component, e.g., volumic number of entities (= number density) $n=N_{\mathrm{S}} / V_{\mathrm{S}}$, molar mass of a compound $M_{\mathrm{B}}=$ $m_{\mathrm{B}} / n_{\mathrm{B}}$, and

- "compositional kind-of-quantity" defined as a ratio between a numerator referring to a component and the denominator referring to the system or to the sum of a set of components, e.g., volume fraction of a component in a system $\varphi_{\mathrm{B}}=V_{\mathrm{B}} / \Sigma V(\mathrm{~A}, \ldots, \mathrm{N})$; amount-ofsubstance content (= substance content [4-9.98.1]) $v_{\mathrm{B}}=n_{\mathrm{B}} / m_{\mathrm{S}}$-not to be confused with the molality of a solute component $b_{\mathrm{B}}=n_{\mathrm{B}} / m_{\mathrm{A}}$, where $\mathrm{A}$ is the solvent; catalytic activity concentration (= catalytic concentration) $b_{\mathrm{B} \text {,cat }}=z_{\mathrm{B}, \mathrm{cat}} / V_{\mathrm{S}}$ where $z_{\mathrm{B} \text {,cat }}$ is catalytic activity of component $\mathrm{B}$.

"Compositional kind-of-quantity" may be divided into:

\footnotetext{
- "fraction" $Q_{\mathrm{B}} / \Sigma Q(\mathrm{~A}, \ldots, \mathrm{N})$

- "content" $Q_{\mathrm{B}} / m_{\mathrm{S}}$,

- "concentration" $Q_{\mathrm{B}} / V_{\mathrm{S}}$,
}

each of which can be further divided into kinds-of-quantity according to the choice of numerator kind-of-quantity.

\section{Concentration}

There are many possible useful divisions of "concentration", obtained by choosing an extensive kind-of-quantity for the numerator. Prominent examples are:

- number concentration $C_{\mathrm{B}}=N_{\mathrm{B}} / V_{\mathrm{S}}$;

- mass concentration $\rho_{\mathrm{B}}=m_{\mathrm{B}} / V_{\mathrm{S}}$ (also symbolized $\gamma_{\mathrm{B}}$ [8]);

- volume concentration $\sigma_{\mathrm{B}}=V_{\mathrm{B}} / V_{\mathrm{S}}$ (is not found in current sources, but should be distinguished from volume fraction $\left.\varphi_{\mathrm{B}}=V_{\mathrm{B}} / \Sigma V(\mathrm{~A}, \ldots, \mathrm{N})\right)$; and

- amount-of-substance concentration (= substance concentration [4-p. 4], amount concentration [2-p. 38], concentration [2-p. 38], previously 'molarity'), $c_{\mathrm{B}}=$ $n_{\mathrm{B}} / V_{\mathrm{S}}$.

The systematic term 'amount-of-substance concentration' is rather unwieldy, begging for clipping. This was achieved 1975 by the clinical chemists in the International Federation of Clinical Chemistry and the International Union of Pure and Applied Chemistry-with the consent of the IUPAC physical chemists-by coining 'substance concentration' [4-p. 4]. Later the physical chemists chose 'amount concentration' or simply 'concentration' [2-p. 38]. In view of the latter term also for the superordinate generic concept described above, this shortest term should only be used after having given the full term and definition. 'Amount concentration' [2-p. 38] is not mentioned by ISO 31 [1-813], but is given in the SI brochure [6-p. 117]. This clipped term has the drawback that 'amount' in ordinary language can also mean number, mass, and volume. The terminological muddle is due to the unfortunate primary choice of the term 'amount of substance' for the base kind-ofquantity, using two nouns with wide meanings in ordinary language [9].

\section{Conclusion}

The current loose use of the term 'concentration' with different meanings, i.e., a case of polysemy, is an example of a potentially harmful practice. By defining each concept and relating them in a concept system (Fig. 1), it is possible to create a systematic nomenclature with monosemy, thus avoiding ambiguity, and increasing information and the understanding of relations. 
Fig. 1 Concept diagram of kind-of-quantity leading to division of "concentration"; heavy lines independent terminological dimensions, light lines generic relations, three dots other possible concept

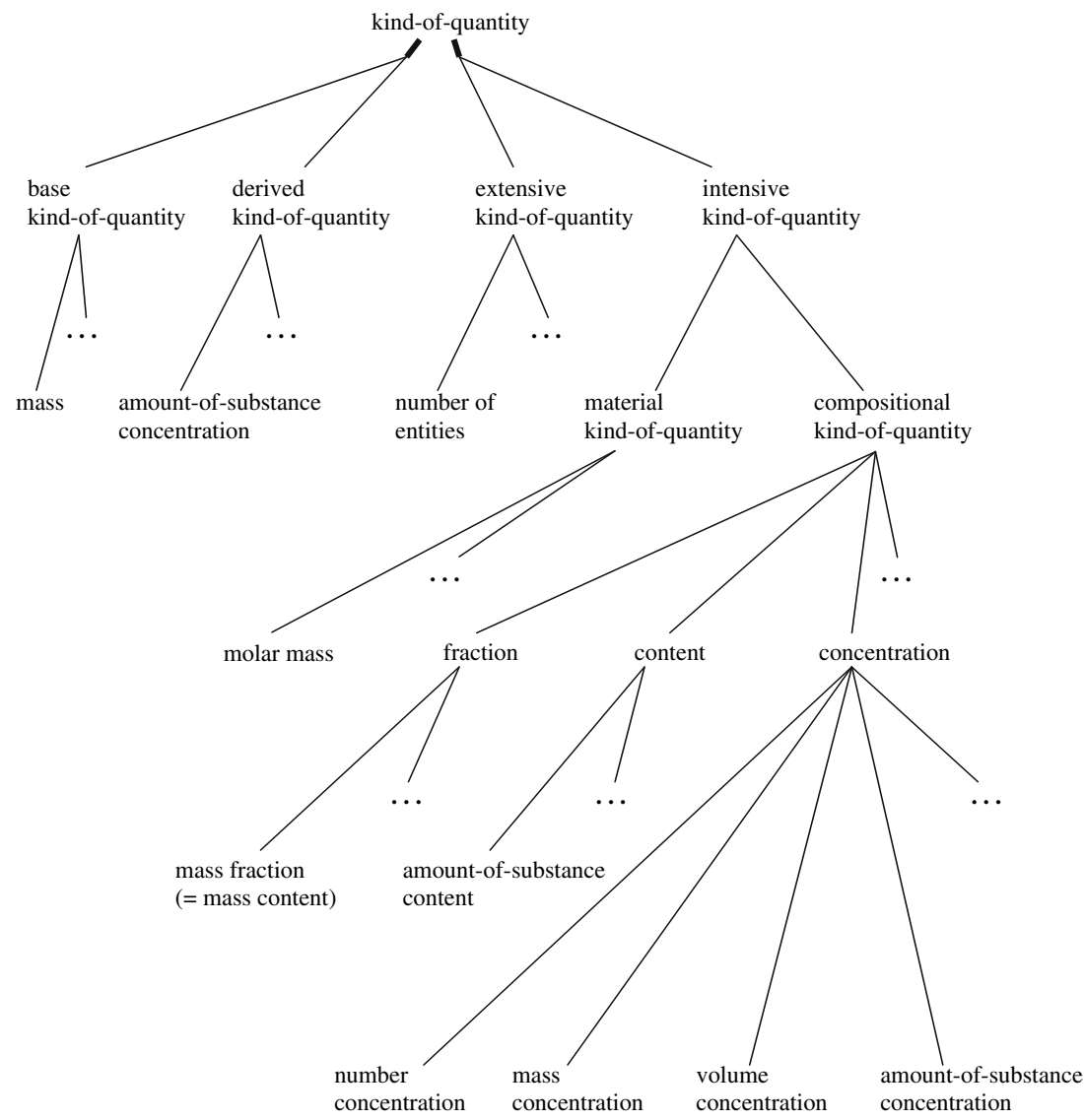

5. Dybkaer R (2004) An ontology for physical, chemical, and biological systems. Thesis APMIS (Acta Path Microbiol Immunol Scand) 122(Suppl 117):1-210

6. BIPM (2006) Le Système international d'unités. The International System of Units, SI. 8th edn. BIPM, Sèvres 1-180

7. BIPM, IEC, IFCC, ILAC, ISO, IUPAC, IUPAP, OIML (2007) International vocabulary of metrology - basic and general concepts and associated terms. Vocabulaire international de métrologie-concepts fondamentaux et généraux et termes associés, 3rd edn. ISO, Geneva (abbreviated VIM3) (in press)

8. Richter W (2007) Recommendations on quantities, symbols and measurement units for publication in ACQUAL. Accred Qual Assur 12:497-498

9. Dybkær R (2000) The term 'chemon' instead of 'amount of substance' allows a succinct systematic terminology and harmonized equivalents in translation. Metrologia 37:301-304 